\title{
Introduction to the Photon Collider
}

\author{
V.I. Telnov \\ Budker Institute of Nuclear Physics, 630090 Novosibirsk, Russia \\ Novosibirsk State University, 630090 Novosibirsk, Russia
}

\begin{abstract}
The purpose of this Introduction, presented at PHOTON2007 1, is to provide an overview of the basic principles, possible parameters, some technical aspects and the physics program of the photon collider and discuss its status within the ILC project.
\end{abstract}

\section{Introduction and politics}

There is a broad agreement within the worldwide particle physics community that the International Linear Collider (ILC) is the most likely and desirable candidate for the next large HEP project. If the LHC discovers new physics accessible to a $0.5-1.0 \mathrm{TeV}$ $e^{+} e^{-}$linear collider, ILC construction can start in 2012-2015. A photon $(\gamma \gamma, \gamma e)$ collider, based on backward Compton scattering of laser light off high-energy electrons, is a natural extension of the linear collider concept 2, 3, 4, 5]. It has been widely argued in the literature that addition of the photon collider to the ILC design would nearly double the ILC physics program, while the total cost of the ILC project would increase only by a few percent.

The history of the photon collider is more than 25 years in the making, as described in detail in my talk at Photon2005 [6]. The photon collider design was developed and refined by enthusiasts in parallel with work on the $e^{+} e^{-}$linear collider; the LC and LCWS workshops and special Photon Collider workshops provided a forum for discussions and brainstorming. About $20 \%$ of papers on linear colliders are devoted to the photon collider (mainly, the physics).

The basic principle of the photon collider is rather simple: it's just Compton scattering. However, it took nearly two decades to gain understanding of the realistic photon-collider performance and to find solutions to the central technical challenges such as removal of disrupted beams from the interaction region, mitigation of backgrounds, collision effects, luminosity optimization, beam dump, requirements to the laser parameters, the optical scheme and laser technologies that allow conversion of almost all electrons to photons, stabilization of beam collisions, measurement of the luminosity, etc. We have now achieved understanding, at a conceptual level, of all critical issues in the design of the photon collider and know how to solve them [7, 8].

The photon collider was considered in the NLC [9], JLC [10, TESLA [11] conceptual design reports and in the TESLA technical design report [7. Motivated to a great extent by the large cost of building a high-energy linear collider, in 2004 the three regional projects were transformed into a single one, the ILC, which would be based on the superconducting technology developed by the TESLA collaboration.

On one hand, unification of the three projects was a productive development as it brought together the expertise of several accelerator laboratories and financial resources of many countries. On the other, as indicated by millenia of human experience in all kinds of endeavors, presence of viable competition nearly always accelerates the rate of progress, while absence of competition can sometimes be detrimental.

It is interesting to consider the ILC schedule in the context of the above observation. In the 1990s, and even at Snowmass 2001, NLC, JLC and TESLA were intended 
not merely as complementary to the LHC, but also as LHC's contemporaries and direct competitors. In fact, TESLA could have started operation in 2010 !

Since 2001, we have seen a dramatic revision of the ILC schedule: 2010 is now the year the ILC Engineering Design is due, with construction beginning in 2012 and completing in 2019 being the most optimistic projection. U.S. DOE officials expect a further delay beyond a technology-driven timeline due to a long process of international negotiations and the need to create the necessary legal framework for the ILC; start of ILC operation in the late 2020s is now being floated.

Recognizing the danger of a long time gap between the scheduled end of Tevatron operation in 2009 and the start of ILC construction, Fermilab, which is considered as the most suitable site for the ILC in the United States, has developed a back-up plan for the case of a prolonged ILC delay, dubbed Project $X$ : a $8 \mathrm{GeV}$ superconducting proton linac for the study of neutrino physics and of help with promising future project (VLHC, muon collider, etc.) If Project $X$ is accepted, a further delay in ILC construction is guaranteed. The question is, would the ILC, as it is envisioned today, still be relevant if it is built on a greatly delayed schedule?

Given the uncertain ability of the United States to host the ILC, a number of alternatives will now be considered: Asia, Europe, Russia. Any construction decision would have to wait for results from the LHC. If new physics below $0.5 \mathrm{TeV}$ is found at $\mathrm{LHC}$, a decision to start ILC construction at one of the proposed sites can be made with little delay.

Let us now review the status of the photon collider within the ILC project. The ICFA Scope document on the ILC refers to $e^{+} e^{-}$ collisions at $2 E=500 \mathrm{GeV}$ as "the baseline", while all other configurations $\left(e^{-} e^{-}\right.$, photon collider, Giga- $Z$, operation at the $W W$ threshold, fixed-target, polarized $e^{+}$beams) are considered "options". At the same time, the ICFA Scope document dictates that the baseline ILC design must be made compatible with the future photon collider. Indeed, to make the photon collider possible in the second stage of the ILC project, it is extremely important from the very start to design the ILC to allow simple transitions between the $e^{+} e^{-}$and $\gamma \gamma$, $\gamma e$ modes of operation when they become available.

Unfortunately, the compatibility with the photon collider was lost in preparation of the ILC Reference Design Report (RDR) 12. Driven by a perceived need to reduce as much as possible the initial ILC cost, the RDR team considered only the basic $e^{+} e^{-}$mode and was a bit too overzealous in cost-cutting. It made the unwise decision to propose a collider with a single IP and a 14 mrad crossing angle, not compatible with the photon collider, which requires a crossing angle of 25 mrad.

While reducing the initial ILC cost by a few percent, the single-IP solution with no space for the photon collider risked a great escalation of the cost of upgrades, to no small part due to the need for substantial additional excavation in the IP region half-way through the ILC lifetime, which would be highly impractical and perhaps technologically or politically impossible. It is obvious that the total cost is minimal when all underground construction work is done at once rather than in two or more stages, with their considerable set-up costs and the disruption they would cause to ILC operation. Gravely concerned with the risks of the single-IP, 14 mrad crossing-angle solution and its near incompatibility with the photon collider, we strongly disagreed with this specific aspect of the RDR and made an effort to change the situation.

And now, good news: shortly after PHOTON07, the GDE agreed that the ILC Engineering Design should include the photon collider. At IRENG07 [13] (September 2007), it was decided to correct the layout of the interaction-region area in order to make it compatible with $\gamma \gamma$ collisions. It would be 
still a single-IP configuration, but underground space will be reserved for an upgrade to the $25 \mathrm{mrad}$ crossing angle. So, the photon collider effort is back on track after two years of uncertainties (and a struggle for its very existence). Indeed, the years 2005-2007 were the most difficult for the photon collider in its $25+$-year history. Now, we can take a breath of fresh air, relax a bit, and continue working towards making the photon collider a reality.

After this positive "political information", I turn to discussing the scientific problems of the photon collider.

\section{Principles and properties of the photon collider}

\subsection{The idea of the photon collider}

Two-photon physics had been talked about since 1930s, but as an active research field is began in early 1970s, when production of $e^{+} e^{-}$pairs was discovered in collisions of virtual photons at an $e^{+} e^{-}$storage ring. In the years that followed many interesting two-photon reactions were studied, but the results could not compete with the revolutionary discoveries made in $e^{+} e^{-}$annihilation. The reason for this is that the luminosity and energy in virtual $\gamma \gamma$ collisions are small. Indeed, the number of equivalent photons surrounding each electron is $d N_{\gamma} \sim 0.035 d \omega / \omega$, and the corresponding $\gamma \gamma$ luminosity for $W_{\gamma \gamma} / 2 E_{0}>0.2$ is only $L_{\gamma \gamma} \approx 4 \times 10^{-3} L_{e^{+} e^{-}}$and an order of magnitude smaller for $W_{\gamma \gamma} / 2 E_{0}>0.5$.

The idea how to achieve much higher $\gamma \gamma$ luminosities was proposed by the author of this paper at the First USSR workshop on the physics at the linear collider VLEPP held in Novosibirsk in December 1980. Here it is in a nutshell: at linear colliders, beams are used only once, which makes it possible to convert electrons to photons, and thus to obtain collisions of real photons. All that is needed is some sort of a target a small distance from the interaction point (IP), where the conversion would take place. For example, if one were to place a target of $0.3 X_{0}$ thickness, the number of bremsstrahlung photons would be greater than the number of virtual photons by one order of magnitude, and the corresponding $\gamma \gamma$ luminosity would increase by two orders of magnitude; however, this approach suffers from photo-nuclear backgrounds. Laser light would make a much better target.

The method of production of high-energy photons by Compton scattering of laser light off high-energy electrons was proposed in 1963 [14 and soon afterwards was tested. However, the conversion coefficient was very small, about $k=N_{\gamma} / N_{e} \sim 10^{-7}$. For the photon collider, we needed $k \sim 1$, seven orders of magnitude more!

Soon after the 1980 VLEPP workshop, a group of $\gamma \gamma$ enthusiasts, I. Ginzburg, G. Kotkin, V. Serbo and V. Telnov, considered the possibility of a photon collider based on the laser conversion. We found that the required flash energy is about 10 J. Extrapolating the progress of laser technologies into the next two decades with a high degree of optimism, we came to the conclusion that a photon collider based on laser photon conversion is not such a crazy idea after all, and in February 1981 published the preprint, and then the paper 2]. Somewhat latter, we published two "thick" papers on this subject [3].

The history of the photon collider and from its origin to the present is described in detail in [6].

\subsection{Basics of the photon collider}

Here, we briefly consider the main characteristics of backward Compton scattering and the requirements on the lasers.

\subsubsection{Kinematics and photon spectra}

In the conversion region, a laser photon of energy $\omega_{0}$ collides with a high-energy electron 
of energy $E_{0}$ at a small collision angle $\alpha_{0}$ (almost head-on). The energy of the scattered photon $\omega$ depends on the photon scattering angle $\vartheta$ with respect to the initial direction of the electron as follows [3]:

$$
\omega=\frac{\omega_{m}}{1+\left(\vartheta / \vartheta_{0}\right)^{2}}
$$

where

$$
\begin{gathered}
\omega_{m}=\frac{x}{x+1} E_{0}, \quad \vartheta_{0}=\frac{m c^{2}}{E_{0}} \sqrt{x+1}, \\
x=\frac{4 E \omega_{0}}{m^{2} c^{4}} \cos ^{2} \frac{\alpha_{0}}{2} \simeq 19\left[\frac{E_{0}}{\mathrm{TeV}}\right]\left[\frac{\mu \mathrm{m}}{\lambda}\right],
\end{gathered}
$$

$\omega_{m}$ being the maximum energy of scattered photons. For example: $E_{0}=250 \mathrm{GeV}, \omega_{0}=$ $1.17 \mathrm{eV}(\lambda=1.06 \mu \mathrm{m})$ (for the most powerful solid-state lasers $) \Rightarrow x=4.5$ and $\omega_{m} / E_{0}=$ 0.82. Formulae for the Compton cross section can be found elsewhere [3, 7].

The energy spectrum of the scattered photons depends on the average electron helicity $\lambda_{e}$ and that of the laser photons $P_{c}$. The "quality" of the photon beam, i.e., the relative number of hard photons, is improved when one uses beams with a negative value of $\lambda_{e} P_{c}$. The energy spectrum of the scattered photons for $x=4.8$ is shown in Fig. 1 for various helicities of the electron and laser beams.

With increasing $x$, the energy of the backscattered photons increases, and the energy spectrum becomes narrower. However, at large values of $x$, photons may be lost due to creation of $e^{+} e^{-}$pairs in collisions with laser photons [3, 4, 5]. The threshold of this reaction is $\omega_{m} \omega_{0}=m^{2} c^{4}$, which corresponds to $x=2(1+\sqrt{2}) \approx 4.83$. One can work above this threshold, but with a reduced luminosity; the luminosity loss factor is in the 5 -10 range for $x=10-20$. Therefore, $x \approx 4.8$ is the most preferable value. The optimum wavelength of the laser photons corresponding to $x=4.8$ is

$$
\lambda=4.2 E_{0}[\mathrm{TeV}] \mu \mathrm{m} .
$$

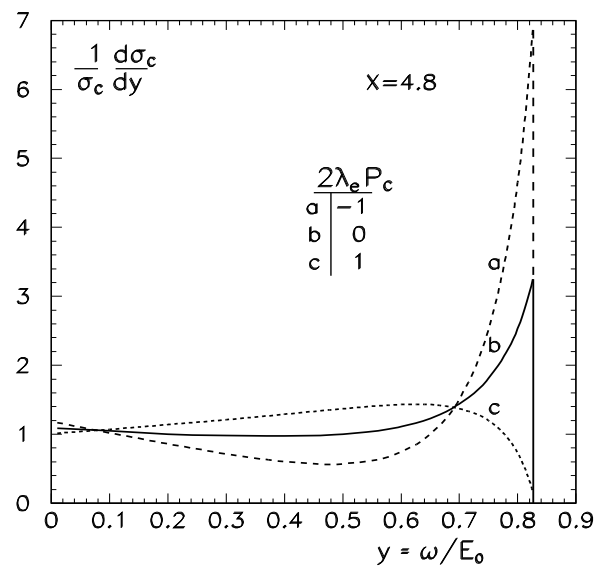

Figure 1: Spectrum of the Comptonscattered photons.

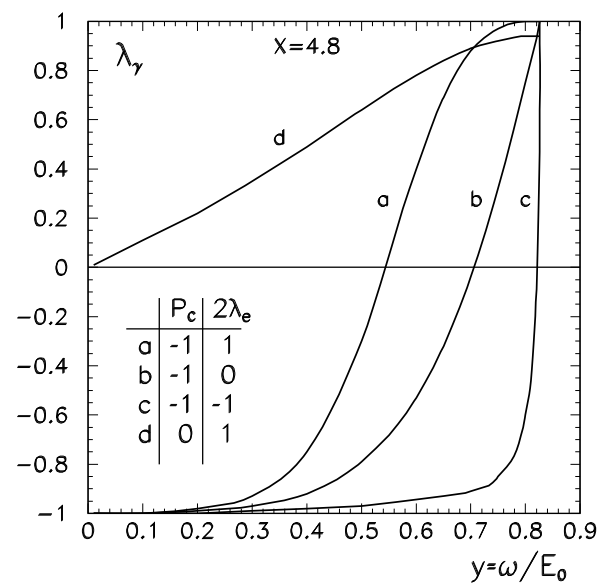

Figure 2: Average helicity of the Comptonscattered photons.

The mean helicity of backscattered photons at $x=4.8$ is shown in Fig. 2 for various helicities of the electron and laser beams. For $2 P_{c} \lambda_{e}=-1$ (the case of the peaked energy spectrum), all photons in the high-energy peak have a high degree of like-sign polarization. A high degree of circular photon polarization is essential for the study of many physics processes, for example, for suppression of QED background in the study of the Higgs boson [7].

Linear polarization of backscattered photons is also possible at the photon collider. 
The degree of the linear polarization at maximum photon energy depends on parameter, it is $l_{\gamma}=0.334,0.6,0.8$ for $x=4.8,2,1$, respectively [7]. The linear polarization is important for the Higgs study .

\subsubsection{Nonlinear effects in the conversion}

The electromagnetic field in the laser wave at the conversion region is very strong, and so electrons can interact with several laser photons simultaneously. These nonlinear effects are characterized by the parameter (see [7] and references therein)

$$
\begin{gathered}
\xi^{2}=\frac{e^{2} \overline{F^{2}} \hbar^{2}}{m^{2} c^{2} \omega_{0}^{2}}=\frac{2 n_{\gamma} r_{e}^{2} \lambda}{\alpha}= \\
=0.36\left[\frac{P}{10^{18} \mathrm{~W} / \mathrm{cm}^{2}}\right]\left[\frac{\lambda}{\mu \mathrm{m}}\right]^{2},
\end{gathered}
$$

where $F$ is the r.m.s. strength of the electric (magnetic) field in the laser wave and $n_{\gamma}$ is the density of laser photons. At $\xi^{2} \ll 1$, the electron scatters on one laser photon, while at $\xi^{2} \gg 1$ multi-photon scattering takes place.

The transverse motion of an electron through the electromagnetic wave leads to the decrease of the maximum energy of the scattered photons: $\omega_{m} / E_{0}=x /\left(1+x+\xi^{2}\right)$. At $x=4.8$, the value of $\omega_{m} / E_{0}$ decreases by about $5 \%$ for $\xi^{2}=0.3$. For plots demonstrating evolution of the Compton spectra as a function of $\xi^{2}$ please refer to Refs. [15, 7]. With increasing $\xi^{2}$, the Compton spectrum is shifted towards lower energies and higher harmonics appear, and the $\gamma \gamma$ luminosity spectra become broader. So, the value of $\xi^{2} \sim 0.3$ can be taken as the limit for $x=4.8$; for smaller values of $x$ it should be even lower.

\subsubsection{Laser flash energy}

While calculating the required flash energy, one must take into account the diffractive divergence of the laser beam and to keep the nonlinear parameter $\xi^{2}$ small. The r.m.s. radius of the laser beam near the conversion region depends on the distance $z$ to the focus (along the beam) as [3]

$$
a_{\gamma}(z)=a_{\gamma}(0) \sqrt{1+z^{2} / Z_{R}^{2}}, a_{\gamma}(0) \equiv \sqrt{\frac{\lambda Z_{R}}{2 \pi}},
$$

where $Z_{R}$ is the Rayleigh length characterizing the length of the focal region determined by focusing optics. Neglecting multiple scattering, the dependence of the conversion coefficient on the laser flash energy $A$ can be written as

$$
k=N_{\gamma} / N_{e} \sim 1-\exp \left(-A / A_{0}\right),
$$

where $A_{0}$ is the laser flash energy for which the thickness of the laser target is equal to one Compton collision length. The value of $A_{0}$ can be roughly estimated from the collision probability $p \sim 2 n_{\gamma} \sigma_{c} \ell=1$, where $n_{\gamma} \sim A_{0} /\left(\pi \omega_{0} a_{\gamma}^{2} \ell_{\gamma}\right), \sigma_{c}$ is the Compton cross section $\left(\sigma_{c}=1.8 \times 10^{-25} \mathrm{~cm}^{2}\right.$ at $\left.x=4.8\right)$, $\ell$ is the length of the region with a high photon density, which is equal to $2 Z_{R}=4 \pi a_{\gamma}^{2} / \lambda$ at $Z_{R} \ll \sigma_{L, z} \sim \sigma_{z}\left(\sigma_{z}, \sigma_{L, z}\right.$ are the r.m.s. lengths of the electron and laser bunches), and the factor 2 is due to the relative velocity of electrons and laser photons. This gives, for $x=4.8$,

$$
A_{0} \sim \frac{\pi \hbar c \sigma_{z}}{2 \sigma_{c}} \sim 3 \sigma_{z}[\mathrm{~mm}], \mathrm{J} .
$$

Note that the required flash energy decreases when the Rayleigh length is reduced to $\sigma_{z}$, but it hardly changes with further decreas$\operatorname{ing} Z_{R}$. This happens because the density of photons grows but the length of the high-density region decreases, and as result the Compton scattering probability remains nearly constant. So, it is not helpful to make the radius of the laser beam at the focus smaller than $a_{\gamma}(0) \sim \sqrt{\lambda \sigma_{z} / 2 \pi}$, which may be much larger than the transverse electron bunch size in the conversion region. From (6) one can see that the flash energy $A_{0}$ is proportional to the electron bunch length, and for $\sigma_{z}=0.3 \mathrm{~mm}$ (ILC) it is about $1 \mathrm{~J}$. The required laser power is about half a terawatt. 
Higher-precision calculations of the conversion probability in head-on collision of a Gaussian laser beam with an electron beam can be found elsewhere [3, 4, 5, 9]; they are close to the above estimate.

However, this is not a complete picture, since one should also take into account the following effects:

- Nonlinear effects in Compton scattering. The photon density is restricted by this effect. For shorter bunches, nonlinear effects will determine the energy of the laser flash.

- Collision angle. If the laser and electron beams do not collide head-on (if the laser optics is outside the electron beam), the required laser flash energy is larger by a factor of $2-2.5$.

- Transverse size of the electron beam. In the crab-crossing scheme, the electron beam is tilted, which leads to an effective transverse beam size comparable to the optimum laser spot size.

Dependence of the $\gamma \gamma$ luminosity on the flash energy and $f_{\#}=F / 2 R$ (flat-top laser beam) for several values of the parameter $\xi^{2}$ is presented in Fig. 3 [16, 8]. This simulation is based on the formula for the field distribution near the laser focus for flat-top laser beams. It was assumed that $\alpha_{c}=25$ mrad and the angle between the horizontal plain and the edge of the laser beam is 17 mrad (the space required for disrupted beams and quads). At the optimum, $f_{\#} \sim 17$, or the angular size of the laser system is about $\pm 0.5 / f_{\#} \approx \pm 30 \mathrm{mrad}$. If the focusing mirror is situated outside the detector at a distance of $15 \mathrm{~m}$ from the IP, it should have a diameter of about $1 \mathrm{~m}$. All other mirrors in the ring cavity can have smaller diameters; about $20 \mathrm{~cm}$ seems sufficient from radiationdamage considerations. This simulation as well as calculations done independently by the Zeuthen group [17, show that with all effects taken into account, the required flash energy for the photon collider at the ILC with $2 E_{0}=500 \mathrm{GeV}$ and for $\lambda=1.05 \mu \mathrm{m}$ is $A \approx 9$ $\mathrm{J}, \sigma_{t} \sim 1.5 \mathrm{ps}, a_{\gamma}(0)=\sqrt{2} \sigma_{\gamma, x} \sim 10 \mu \mathrm{m}$. The corresponding peak power is $2.5 \mathrm{TW}$.

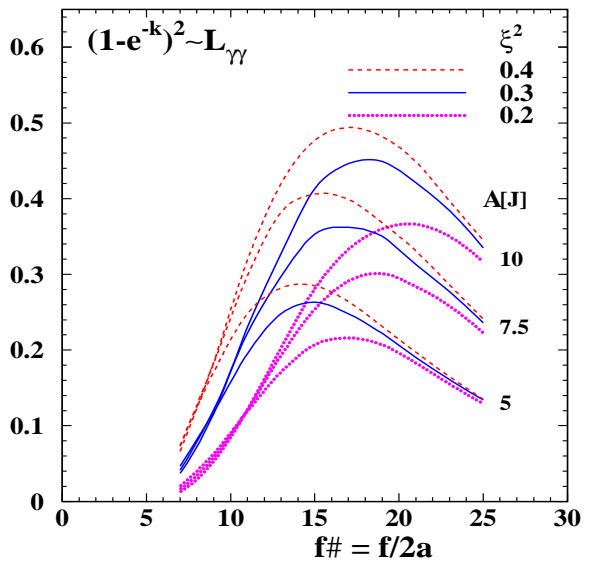

Figure 3: Dependence of $L_{\gamma \gamma}$ on the flash energy and $f_{\#}$ (flat-top laser beam) for several values of the parameter $\xi^{2}$. See comments in the text.

The same laser with the $1 \mu \mathrm{m}$ wavelength can be used up to an ILC energy $2 E_{0} \sim 700$ $\mathrm{GeV}$ [16, 8]. At higher energies, the $\gamma \gamma$ luminosity decreases due to $e^{+} e^{-}$pair creation in the conversion region in collisions of the highenergy and laser photons and due to the decrease of the Compton cross section. For the ILC energy $2 E_{0}=1.0 \mathrm{TeV}$, the reduction in the luminosity due to these effects is about a factor of 2-3 compared to the optimum case. For $2 E_{0}=0.7-1 \mathrm{TeV}$, it is desirable to have a wavelength of about $1.5-2 \mu \mathrm{m}$. The technical feasibility of such a laser has not been studied yet.

\section{$3 \quad$ Interaction region issues}

\subsection{Collision scheme}

The general scheme of the photon collider is shown in Fig. 4. The laser light is focused on the electron beam in the conversion region $\mathrm{C}$, at a distance of $b \mathrm{~cm}$ from the interaction point IP; after Compton scattering, the high-energy photons follow along the initial electron trajectories with a small additional 
angular spread $\sim 1 / \gamma$, i.e., they are in focus at the interaction point IP. Thus obtained $\gamma$ beam collides downstream either with an oppositely directed electron beam or another $\gamma$ beam.
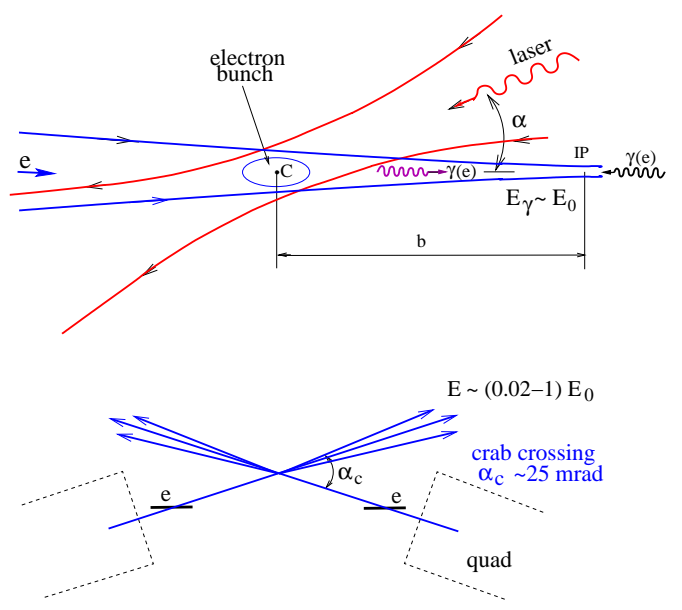

Figure 4: Scheme of $\gamma \gamma, \gamma e$ collider.

In the originally proposed scheme [2, 3], following the conversion the electrons were swept away by a magnetic field $B \sim 1 \mathrm{~T}$. The scheme with magnetic deflection of used beams allowed rather clean $\gamma \gamma$ or $\gamma e$ collisions to be produced. Taking $b \gg \gamma a_{e}$, one can obtain a $\gamma \gamma$ luminosity spectrum with a width of $\sim 10-15 \%$ (the "monochromatization effect" 3). The optimum distance $b$ corresponds to the case when the size of the photon beams at the IP due to Compton scattering is comparable to the vertical (minimum) size of the electron beam: $b \sim \sigma_{y} \gamma$. For the first linear collider projects, VLEPP and SLC, this distance was about $10 \mathrm{~cm}$, which was sufficient for magnetic deflection.

A year later, the vertical beam sizes in LC projects under consideration were revised down to $3-5 \mathrm{~nm}$. For $\sigma_{y}=3 \mathrm{~nm}$, the opti$\operatorname{mum} b \sim \gamma \sigma_{y} \sim 1.5 \mathrm{~mm}$ for $2 E_{0}=500 \mathrm{GeV}$. This space is too small to fit any kind of a magnet. Therefore, since 1991 [18] we have been considering a scheme with no magnetic deflection. In this case, there is a mixture of $\gamma \gamma, \gamma e$ and $e^{-} e^{-}$collisions, beamstrahlung photons give a very large contribution to the $\gamma \gamma$ luminosity at the low and intermediate invariant masses, the backgrounds are larger, and the disruption angles are larger than in the scheme with magnetic deflection (due to deflection of low-energy particles in the field of the on-coming beam). However, there are certain advantages: the scheme is simpler, and the luminosity is larger. As for the backgrounds, they are larger but tolerable.

Note that even in the absence of deflecting magnets there is the beam-beam deflection, which suppress the residual $e^{-} e^{-}$luminosity. Also, at large CP-IP distances and a nonzero crossing angle, the detector field serves as the deflecting magnet and allows more-orless clean and quite monochromatic $\gamma \gamma, \gamma e$ collisions to be obtained with a reduced luminosity, which will be useful for QCD studies [19].

\subsection{The removal of beams}

After crossing the conversion region, the electrons have a very broad energy spectrum, $E=(0.02-1) E_{0}$, and so the removal of such a beam from the detector is far from trivial. In the scheme with magnetic deflection, all charged particles travel in the horizontal plane following the conversion. At the IP, they get an additional kick from the oncoming beam, also in the horizontal plane. This gave us a hope that the beams can be removed through a horizontal slit in the final quadrupoles. However it was not clear how to remove beams in the scheme with no magnetic deflection.

In 1988, R. Palmer suggested the crabcrossing scheme for $e^{+} e^{-}$collisions at the NLC in order to suppress the multi-bunch instabilities, Fig. 4 (bottom). In the crabcrossing scheme, the beams are collided at a crossing angle $\alpha_{c}$. In order to preserve the luminosity, the beams are tilted by a special cavity by the angle $\alpha_{c} / 2$. This scheme solves 


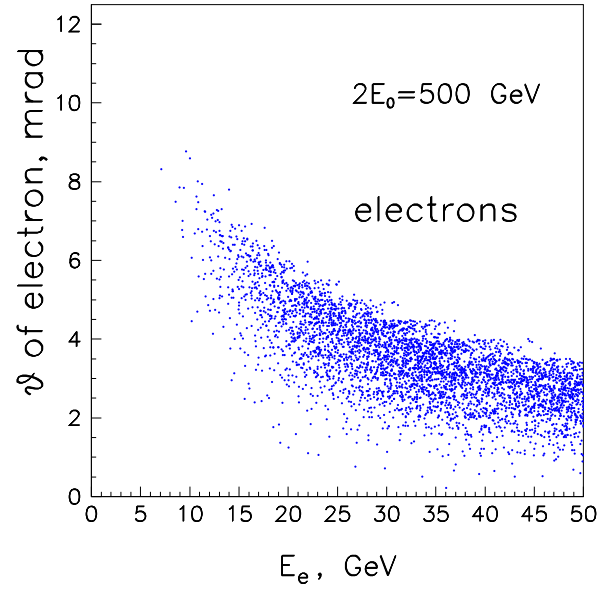

Figure 5: Angles of disrupted electrons after Compton scattering and interaction with the opposing electron beam; $N=2 \times 10^{10}, \sigma_{z}=$ $0.3 \mathrm{~mm}$.

the problem of beam removal at photon colliders [4, 5: the disrupted beams just travel straight outside the quadrupoles.

In the scheme without magnetic deflection (which is now the primary scheme), the low-energy particles get quite a large deflection in the field of the opposing beam. The disrupted beams have an angular spread of about $\pm 10 \mathrm{mrad}$ (12 mrad with tails) after the IP 7, 16, 8, see Fig. 5] The disruption angle for low-energy particles is proportional to $\sqrt{N / \sigma_{z} E}$ [4, 5] and depends very weakly on the transverse beam sizes.

The required crossing angle is determined by the disruption angle, the outer radius of the final quadrupole (about $5 \mathrm{~cm}$ [16, 8]), and the distance between the first quad and the IP (about $4 \mathrm{~m}$ ), which gives $\alpha_{c}=12+$ $5 / 400 \approx 25 \mathrm{mrad}$.

\subsection{The layout of the photon collider at the ILC}

For many years it was assumed that the future linear collider would have two IPs, each equipped with a detector, where the second
IP, with a larger crossing angle, would be optimized for the photon collider. In the first few years, both detectors would run in the $e^{+} e^{-}$mode. Then, one of the IPs and the detector would be modified for operation in the $\gamma \gamma$, $\gamma e$ mode.

However, as was discussed in the Introduction, in the present ILC design [12] only one IP is planned, with a crossing angle of 14 mrad and two detectors in the pull-push configuration that could be swapped in and out of the interaction region. This is the minimum crossing angle that allows the outgoing beams in $e^{+} e^{-}$collisions to travel outside the quads. On the other hand, at the photon collider the crossing angle should be at least 25 mrad. This creates a problem.

A crossing angle greater than $30 \mathrm{mrad}$ is not desirable because the vertical beam size at the IP would increase due to synchrotron radiation in the detector field. Yet at 25 mrad, the reduction of the luminosity would be very small both for the $e^{+} e^{-}$and $\gamma \gamma, \gamma e$ modes of operation [20, 16]. At first sight, it would therefore seem quite reasonable to design the ILC with 25 mrad crossing angle both for the $e^{+} e^{-}$and the photon collider. Indeed, why not? There are two arguments against it.

First of all, a smaller crossing angle is somewhat better for the study of certain SUSY production processes in $e^{+} e^{-}$collisions where detection of particles at small polar angles is needed for suppression of the Standard Model backgrounds. Then again, the difference between 14 and 25 mrad crossing angles is not that great.

The second, more serious contradiction between $e^{+} e^{-}$and $\gamma \gamma$ has to do with the difference in the requirements on the extraction lines and beam dumps due to the very different properties of electron and photon beams.

In the $e^{+} e^{-}$case, after collision the beams remain quite monochromatic and there is a possibility to measure their properties (the energy spectrum and polarization). Such an extraction line should be quite long and 
equipped with many magnetic elements and diagnostics.

At the photon collider, the situation is different:

- The disrupted beams at a photon collider consist of an equal mixture of electrons and photons (and some admixture of positrons);

- Low-energy particles in the disrupted beams have a large angular spread and need exit pipes of a large diameter.

- Following the Compton scattering, the photon beam is very narrow, with a power of about $10 \mathrm{MW}$. It cannot be dumped directly at any solid or liquid material.

There exists an idea of a beam dump for the photon collider, as well as some simulations [21, 16, 8]. In short, it is a long tube, the first $100 \mathrm{~m}$ of which is vacuum, followed by a $150 \mathrm{~m}$ long gas converter ending in a water-filled beam dump (Fig. 6). The diameter of the tube at the beam dump is about $1.5 \mathrm{~m}$. In addition, there are fast sweeping magnet for electrons. Due to a large beam width, no detailed diagnostics are possible, except perhaps beam profile measurements.

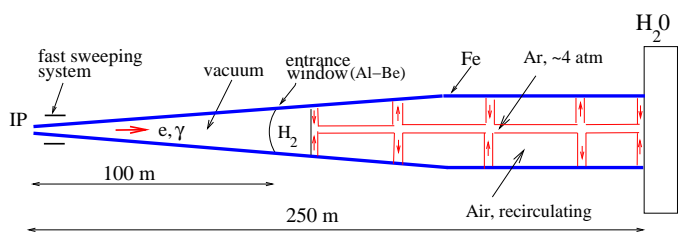

Figure 6: An idea for the photon collider beam dump.

So, the extraction lines and the beam dump for $e^{+} e^{-}$and $\gamma \gamma$ are very different. Their replacement (transition to $\gamma \gamma$ and back after the energy upgrade) would be problematic due to induced radioactivity. Therefore it makes sense to have different crossing angles and separate extraction lines and beam dumps for $e^{+} e^{-}$and $\gamma \gamma$. The suggestion of the ILC beam delivery group at LCWS06 was the following [22]. For the transition from $e^{+} e^{-}$to $\gamma \gamma$, one has to move the detector and about $700 \mathrm{~m}$ of the up-stream beamline, Fig. 7. The displacement of the detector required for the increase of the crab-crossing angle from 14 to $25 \mathrm{mrad}$ is about $4 \mathrm{~m}$. The photon collider would also need an additional $250 \mathrm{~m}$ of tunnels for the beam dumps.

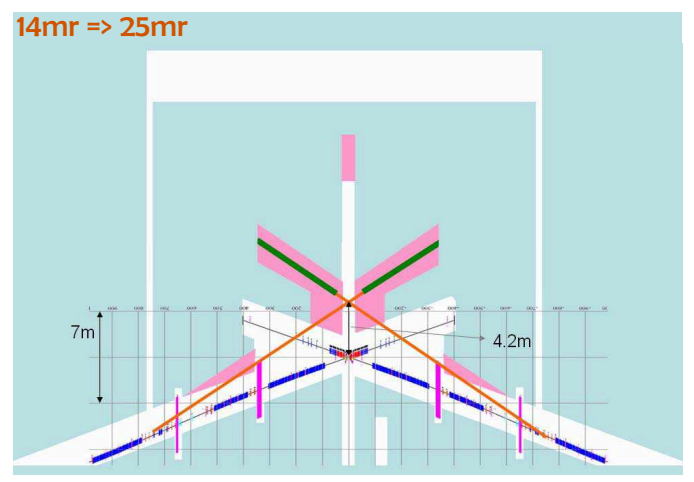

Figure 7: The upgrade path from $e^{+} e^{-}$to $\gamma \gamma$ (14 mrad to $25 \mathrm{mrad}$ )

Such an upgrade would not be easy but in principle is acceptable, provided that all excavation required for the photon collider is done at one time the initial ILC stage is built and the procedure of the detector and beamline displacement is developed in advance.

In my opinion, expressed also at LCWS06 23, the presence of complicated diagnostic devices in the $e^{+} e^{-}$extraction lines is not obligatory. Indeed, even without such special extraction lines we can measure the beam energies and polarizations before collisions, many relevant quantities can be measured during the collision (acollinearity angles, distributions of the secondary $e^{+} e^{-}$ pairs, the beam deflection angles); we can also measure the angular distributions and the charged and neutral contents in the disrupted beams. All this allows the 
reconstruction of the dynamics of beam collisions, with proper corrections applied to the simulation. So, if one were to abandon the idea of instrumented extraction lines for $e^{+} e^{-}$, the crossing angle, beam dumps and beamlines for $e^{+} e^{-}$and $\gamma \gamma$ could be the same, and the upgrade from $e^{+} e^{-}$to $\gamma \gamma$ would be much easier.

The above suggestion was met by the advocates of the special, instrumented $e^{+} e^{-}$extraction lines "without enthusiasm". For the sake of a consensus, I accepted [23] the upgrade path from 14 to 25 mrad proposed by the ILC beam delivery group.

The $\gamma \gamma$ collider and other "options" are discussed in the Physics and Detector volumes of the ILC RDR. On the other hand, the Accelerator volume of the ILC RDR considered only the "baseline" configuration, making no mention of the upgrades and focusing on a considerable reduction of the initial ILC cost, of which the $\gamma \gamma$ collider is only a small part. The intent was to reduce the cost without affecting the overall ILC physics program, and it was thought that the extra excavation required for the photon collider can be done at a later time. As explained above, this is not so, and therefore the photon-collider community could not possibly have been in in agreement with this opinion 24, 25].

Fortunately, common sense has prevailed. The next step in the ILC GDE is the Engineering Design Report. At the ILC Interaction region engineering design workshop, IRENG07 13, we once again formulated the requirements to the ILC design imposed by the upgrade path to the photon collider [26], and they were accepted. The GDE team agreed that the baseline ILC configuration should be modified in order to make it compatible with the photon collider option, and all underground excavation work the photon collider would require should be done from the very beginning.

\subsection{The luminosity}

In $e^{+} e^{-}$collisions, the maximum achievable luminosity is determined by beamstrahlung and beam instabilities. At photon colliders, the only effect that restricts the $\gamma \gamma$ luminosity is the conversion of the high-energy photons into $e^{+} e^{-}$pairs in the field of the opposing beam, that is, coherent pair creation [27, 4]. The threshold for this effect $\kappa=$ $\left(E_{\gamma} / m c^{2}\right)\left(B / B_{0}\right) \sim 1$, where $B_{0}=\alpha e / r_{e}^{2}=$ $4.4 \times 10^{13}$ Gauss is the Schwinger field and $B$ is the beam field. For $\gamma e$ collisions, the luminosity is determined by beamstahlung, coherent pair creation and the beam displacement during the collision.

It is interesting to note that at the centerof-mass energies below $0.5-1 \mathrm{TeV}$ and for electron beams that are not too short, coherent pair creation is suppressed due to the broadening and displacement of the electron beams during the collision [28]: the beam field becomes lower than the threshold for $e^{+} e^{-}$production. So, one can even use infinitely narrow electron beams.

All these processes, and a few others, were included in simulation codes for beam collisions at linear colliders. Results, presented below, were obtained by the code [5], which was used for optimization of the photon colliders at NLC [9] and TESLA [11, 7].

Simulated values of $\gamma \gamma$ and $\gamma e$ luminosities (in the high energy peak) for TESLA (and, similarly, for ILC) are shown in Fig. 8 , 40, 29, 7]. This figure shows how the luminosity depends on the horizontal beam size (the vertical size is much smaller). One can see that all $\gamma \gamma$ luminosity curves follow their natural behavior: $L \propto 1 / \sigma_{x}$. Note that for $e^{+} e^{-}$, the minimum horizontal beam size restricted by beamstrahlung is about $500 \mathrm{~nm}$, while the photon collider can work even with $\sigma_{x} \sim 10$ $\mathrm{nm}$ at $2 E_{0}=500 \mathrm{GeV}$, delivering a luminosity that is several times higher than that in $e^{+} e^{-}$collisions! In fact, the $\gamma \gamma$ luminosity is simply proportional to the geometric $e^{-} e^{-}$ luminosity. 


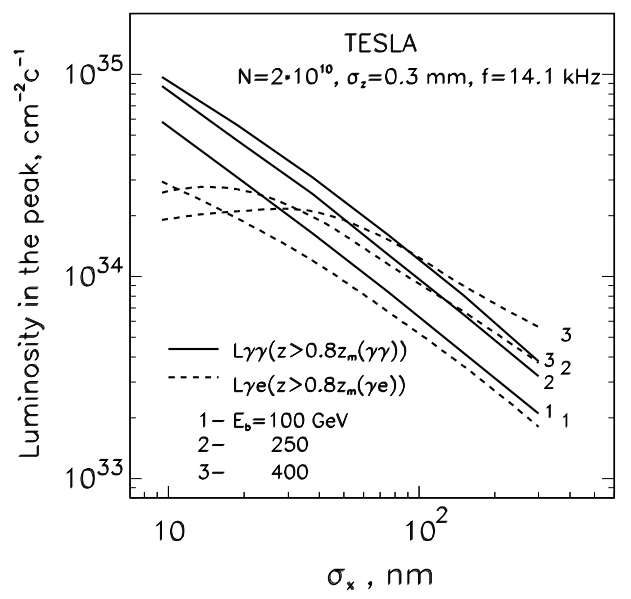

Figure 8: Dependence of $\gamma \gamma$ and $\gamma e$ luminosities in the high energy peak on the horizontal beam size for TESLA-ILC at various energies.

Unfortunately, the beam emittances in the damping-ring designs currently under consideration cannot achieve beam sizes that are smaller than $\sigma_{x} \sim 250 \mathrm{~nm}$ and $\sigma_{y} \sim 5 \mathrm{~nm}$, though a reduction of $\sigma_{x}$ by a factor of two seems possible. In principle, one can use electron beams directly from low-emittance photo-guns, avoiding the need for damping rings altogether, but at present they offer a product of the transverse emittances that is noticeably larger than can be obtained with damping rings (note: the beams should be polarized).

To further reduce the beam emittances downstream of the damping rings or photoguns, one can use the method of laser cooling of the electron beams [30, 31. This method opens the way to emittances that are much lower than those obtainable at damping rings - however, this method requires a laser system that is much more powerful than the one needed to achieve the $e \rightarrow \gamma$ conversion. So, laser cooling of electron beams at linear colliders is a technology for use at $\gamma \gamma$ factories in the distant future.

There is an approximate general rule: the luminosity in the high-energy part of the spectrum $L_{\gamma \gamma} \sim 0.1 L_{\text {geom }}$ [7, where $L_{\text {geom }}=$ $N^{2} \nu \gamma / 4 \pi \sqrt{\epsilon_{n x} \epsilon_{n y} \beta_{x} \beta_{y}}$. In order to maximize the luminosity, one needs the smallest beam emittances $\epsilon_{n x}, \epsilon_{n y}$ and beta-functions at the IP, approaching the bunch length. Compared to the $e^{+} e^{-}$case, where the minimum transverse beam sizes are determined by beamstrahlung and beam instability, the photon collider needs a smaller product of horizontal and vertical emittances and a smaller horizontal beta-function.

The "nominal" ILC beam parameters are: $N=2 \times 10^{10}, \sigma_{z}=0.3 \mathrm{~mm}, \nu=14100$ $\mathrm{Hz}, \epsilon_{n x}=10^{-5} \mathrm{~m}, \epsilon_{n y}=4 \times 10^{-8} \mathrm{~m}$. Obtaining $\beta_{y} \sim \sigma_{z}=0.3 \mathrm{~mm}$ is not a problem, while the minimum value of the horizontal $\beta$-function is restricted by chromogeometric aberrations in the final-focus system 7 . For the above emittances, the limit on the effective horizontal $\beta$-function is about $5 \mathrm{~mm}$ 16, 32. The expected $\gamma \gamma$ luminosity $L_{\gamma \gamma}\left(z>0.8 z_{m}\right) \sim 3.5 \times 10^{33} \mathrm{~cm}^{-2} \mathrm{~s}^{-1}$ $\sim 0.17 L_{e^{+} e^{-}}$(here the nominal $L_{e^{+} e^{-}}=$ $\left.2 \times 10^{34} \mathrm{~cm}^{-2} \mathrm{~s}^{-1}\right)$ [16.

The typical $\gamma \gamma$, $\gamma e$ luminosity spectra for the TESLA-ILC(500) parameters are shown in Fig. 9 (7). They are decomposed to states with different spins $J_{z}$ of the colliding particles. The total luminosity is the sum of the two spectra. The residual $e^{-} e^{-}$luminosity (not shown) is one order of magnitude smaller due to beam repulsion. The luminosities with the cut on the parameter $R$ are added just to show that the low-mass luminosities are due to the very asymmetric collisions. One can see that $\gamma \gamma$ and $\gamma e$ luminosities are comparable and these processes can be studied simultaneously.

However, it is much better to study $\gamma e$ collision when only one of the electron beams is converted to photons. In this case, one can measure the $\gamma e$ luminosity much more precisely 33 and with smaller backgrounds. The problem of measuring the $\gamma e$ luminosity spectra when both beams are converted to photons (not completely) is due to the uncer- 
tainty which direction the photon came from.
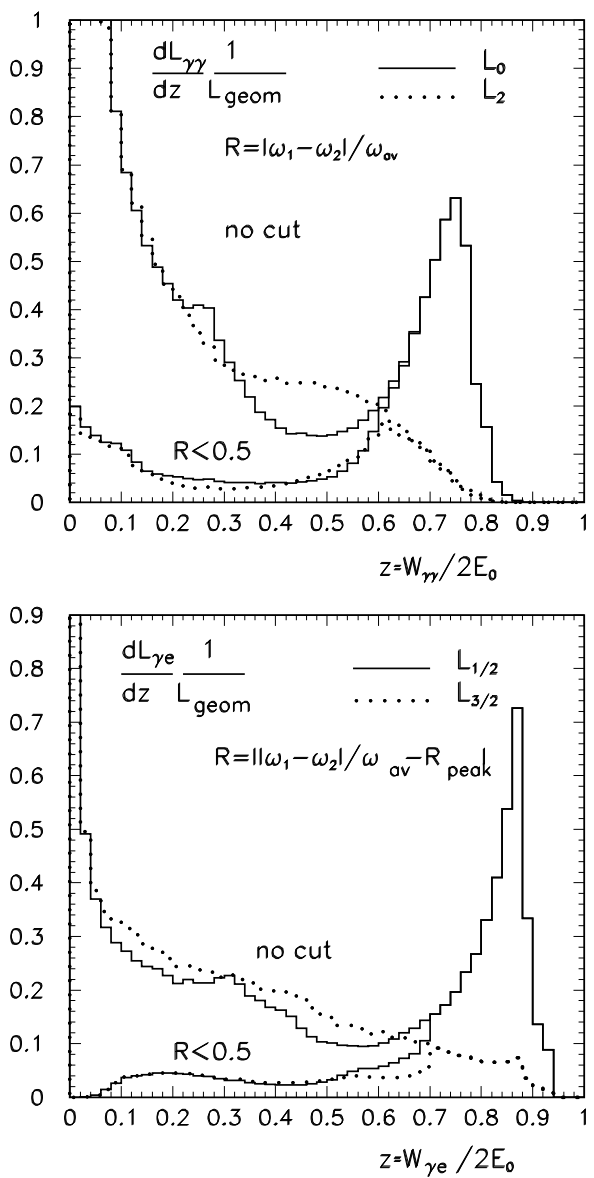

Figure 9: The $\gamma \gamma$ (upper) and $\gamma e$ (bottom) luminosity spectra for typical TESLA (ILC) parameters at $2 E_{0}=500 \mathrm{GeV}$. Solid lines for $J_{z}$ of two colliding photons equal to 0 , dotted lines for $J_{z}=2(1 / 2$ and $3 / 2$, respectively, in the case of $\gamma e$ collisions).

Taking into account the fact that cross sections for many interesting processes are larger in $\gamma \gamma$ collisions than those in $e^{+} e^{-}$by an order of magnitude, the event rate in $\gamma \gamma$ collisions with nominal ILC beams would be similar, or perhaps somewhat larger, than in $e^{+} e^{-}$collisions.

However, it is a highly unsatisfying situation to have the $\gamma \gamma$ luminosity limited by the beam emittances, an order of magnitude below its physics limit (determined by collision effects)! Being able to achieve the physics-limited $\gamma \gamma$ luminosity would open new physics possibilities, such as the study of Higgs self-coupling in $\gamma \gamma$ collisions just above the $\gamma \gamma \rightarrow h h$ threshold 34 .

It is an extremely interesting task to search for a realistic technical solution for obtaining beams with smaller emittances. There are a few good ideas, such as laser cooling, but the first order of business should be trying to optimize the damping rings for the specific requirements of achieving the highest possible luminosity at the photon collider. The importance of optimizing the damping ring design before the start of ILC construction has been emphasized in [16, 8, 35], the damping-ring experts and GDE management are aware of it, but, unfortunately, it appears that up to now the ILC damping-ring design has been guided only by the baseline $e^{+} e^{-}$ considerations.

\subsubsection{Luminosity stabilization}

Beam collisions (luminosity) at linear colliders can be adjusted by a feedback system that measures the beam-beam deflection using beam position monitors (BPM) and corrects beam positions by fast kickers. This method is considered for $e^{+} e^{-}$collisions and is assumed for $\gamma \gamma$ as well [7, 8].

There are some differences between the $e^{+} e^{-}$and $\gamma \gamma$ cases. In the $e^{+} e^{-}$case, at small vertical displacements the beams attract each other and oscillate. In the $\gamma \gamma$ case $\left(e^{-} e^{-}\right.$as well $)$, the beams repel each other; as a result, the deflection angle is larger and almost independent of the initial displacement. There are also some other differences connected with fluctuation of the conversion efficiency. This problem and a stabilization algorithm were considered in detail in Ref. 8 . 


\subsubsection{Luminosity measurement}

The measurement of the luminosity at the photon collider is not an easy task. The spectra are broad and one should measure the luminosity and polarization as a function of energies $E_{1}, E_{2}$ of the colliding particles 33. The luminosity spectrum and polarization can be measured using various QED processes. These are $\gamma \gamma \rightarrow l^{+} l^{-}(l=$ $e, \mu)$ [36, 7, 33], $\gamma \gamma \rightarrow l^{+} l^{-} \gamma$ [33, 37] for $\gamma \gamma$ collisions and $\gamma e \rightarrow \gamma e$ and $\gamma e \rightarrow e^{-} e^{+} e^{-}$for $\gamma e$ collisions 33. Some other SM processes could be useful as well.

\section{The laser and optics}

The photon collider at $\operatorname{ILC}(500)$ requires a laser system with the following parameters: flash energy $A \sim 10 \mathrm{~J}, \sigma_{t} \sim 1.5 \mathrm{ps}, \lambda \sim 1 \mu \mathrm{m}$, and the following ILC pulse structure: 3000 bunches within a $1 \mathrm{~ms}$ train and $5 \mathrm{~Hz}$ repetition rate for the trains, the total collision rate being $15 \mathrm{kHz}$.

In 1981, when the photon collider was proposed, the short-pulse Terawatt lasers required for by a photon collider were just a dream. A breakthrough in laser technologies, the invention of the chirped pulse amplification (CPA) technique [38, occurred very soon, in 1985. The main problem in obtaining short pulses was the limitation of the peak power imposed by the nonlinear refractive index of the medium. This limit on intensity is about $1 \mathrm{GW} / \mathrm{cm}^{2}$; the CPA technique successfully overcame it.

The principle of CPA is as follows. A short, $\sim 100$ fs low-energy pulse is generated in an oscillator. Then, this pulse is stretched by a factor of $10^{4}$ by a pair of gratings, which introduces a delay that is proportional to the frequency. This several-nanosecond-long pulse is amplified, and then compressed by another pair of gratings into a pulse of the initial (or somewhat longer) duration. As nonlinear effects are practically absent in the stretched pulses, the laser pulses obtained with the CPA technique have a quality close to the diffraction limit. This technique now allows the production of not merely $\mathrm{TW}$, but even PW laser pulses, and in several years the Exawatt level will be reached, see the graph of laser power vs time in [6].

The next, very serious problem was the laser repetition rate. The pumping efficiency of traditional flash lamps is very low; the energy is spent mainly on heating the laser medium. In addition, the lifetime of flash lamps is too short, less than $10^{6}$ shots. Semiconductor diode lasers solved these problems. The efficiency of diode laser pumping is very high, and heating of the laser medium is low. The lifetime of the diodes is sufficient for the photon collider.

In addition to the average repetition rate, the time structure is of great importance. The average power required of each of the two lasers for the photon collider at the ILC is $10 \mathrm{~J} \times 15000 \mathrm{~Hz} \sim 150 \mathrm{~kW}$; however, the power within the $1 \mathrm{msec}$ train is $10 \mathrm{~J}$ $\times 3000 / 0.001 \sim 30$ MW! The cost of diodes is about $\mathcal{O}(1 \$) / \mathrm{W}$, the pumping efficiency about $25 \%$, so the cost of just the diodes would be at least $\mathcal{O} \$ 100 \mathrm{M})$, and the size of the facility would be very large.

Fortunately, there is a solution. A $10 \mathrm{~J}$ laser bunch contains about $10^{20}$ laser photons, only about $10^{11}$ of which are knocked out in a collision with the electron bunch. So, it is natural to use the same laser bunch multiple times. There are at least two ways to achieve this: an optical storage ring and an external optical cavity.

In the first approach, the laser pulse is captured into a storage ring using thin-film polarizers and Pockels cells [9, 29, 17. However, due to the nonlinear effects that exist at such powers, it is very problematic to use Pockels cells or any other materials inside such an optical storage ring.

Another, more attractive approach, is an "external" optical cavity that is pumped by a laser via a semi-transparent mirror [39, 40, 41, 29, 17, 42, 17. One can create inside such 
a cavity a light pulse with an intensity that is by a factor of $Q$ (the quality factor of the cavity) greater than the incoming laser power. The value of $Q$ achievable at such powers is $100-200$. This means reduction of the required laser power by a factor of hundred: for obtaining $10 \mathrm{~J}$ in the conversion region one can use a laser with $0.1 \mathrm{~J}$ laser flash and pulse structure similar to the ILC. The optical-cavity principle is illustrated in Fig. 10. The cavity should also include adaptive mirrors and other elements for diagnostic and adjustment.

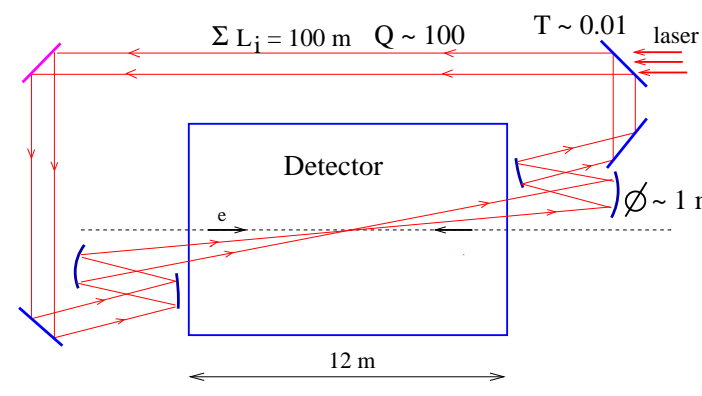

Figure 10: External optical ring cavity for the photon collider

Actually, such external cavities were known long ago in optical laboratories, were used in a FEL experiments, in the gravitational-wave experiment LIGO, etc., but were practically unknown to HEP community: I arrived at this idea in 1999 39. 40. 41 from the first principles, and only later found that this technique already exists. Only then did I finally begin to believe in the technical feasibility of the photon collider with the TESLA-ILC pulse structure and started to push it vigorously [29, 43, 7]. Now, optical cavities are the baseline approach for the laser system at the ILC.

Note that the external optical cavity idea has proven to be a highly useful technique for HEP. It is now used for beam diagnostics ("laser wire"), for production of polarized positrons for linear colliders (see Zomer's talk at this conference 44) and can be used for laser cooling of electron beams [30, 31.

Advancements in laser technologies are mostly driven by a handful of large, well-funded programs, such as inertialconfinement fusion. These technologies are: the chirped-pulse technique, diode pumping, laser materials with high thermal conductivity, adaptive optics (deformable mirrors), disk amplifiers with gas (helium) cooling, large Pockels cells, polarizers, highpower and high-reflectivity multi-layer dielectric mirrors; anti-reflection coatings, etc. Now, practically all laser technologies and components required for a photon collider are in existence; nevertheless, the construction of such a state-of-the-art laser system would not be an easy task.

\section{Physics}

Although the $\gamma \gamma$ luminosity in the highenergy part of the spectrum will be lower than in $e^{+} e^{-}$by a factor of $3-5$, the cross sections in $\gamma \gamma$ collisions are typically greater by a factor of 5-10 [7, so the number of "interesting" events would surpass that in $e^{+} e^{-}$ collisions. Moreover, a further increase of the achievable $\gamma \gamma$ luminosity by up to one order of magnitude may be possible.

Since the photon couples directly to all fundamental charged particles-leptons, quarks, $W$ 's, supersymmetric particles, etc. - the photon collider provides a possibility to test every aspect of the Standard Model, and beyond. Besides, photons can couple to neutral particles (gluons, Z's, Higgs bosons, etc.) through higher-order diagrams.

Many theorists took part in the development of the physics program for the photon collider; the total number of publications has surpassed the 1000 mark.

The physics program at the photon collider would be very rich and complement in an essential way the physics in $e^{+} e^{-}$collisions under any physics scenario. In $\gamma \gamma, \gamma e$ collisions, compared to $e^{+} e^{-}$,

- the energy is smaller only by $10-20 \%$; 
- the number of interesting events is similar or greater;

- access to higher particle masses (single resonances in $H, A$, etc., in $\gamma \gamma$, heavy charged and light neutral (SUSY, etc.) in $\gamma e$ );

- at some SUSY parameters, heavy $H / A$ bosons will be seen only in $\gamma \gamma$;

- higher precisions for some phenomena;

- different types of reactions;

- highly polarized photons.

Some list of gold-plated processes is presented in Table 5. More about physics at

\section{Reaction}

$\gamma \gamma \rightarrow h^{0} \rightarrow b \bar{b}$ $\gamma \gamma \rightarrow h^{0} \rightarrow W W\left(W W^{*}\right) 140<M_{h^{0}}<190 \mathrm{GeV}$ $\gamma \gamma \rightarrow h^{0} \rightarrow Z Z\left(Z Z^{*}\right) \quad 180<M_{h^{0}}<350 \mathrm{GeV}$ $\gamma \gamma \rightarrow h^{0} \rightarrow \gamma \gamma \quad M_{h^{0}}<150 \mathrm{GeV}$ $\gamma \gamma \rightarrow H, A \rightarrow b \bar{b}$ $\gamma \gamma \rightarrow H \tau^{+} \tau^{-} \quad \tan \beta$ in SUSY sector $M S S M$ heavy Higgs $\gamma \gamma \rightarrow \tilde{f} \overline{\tilde{f}}, \tilde{\chi}_{i}^{+} \tilde{\chi}_{i}^{-}, H^{+} H^{-} \quad$ SUSY particles $\gamma \gamma \rightarrow S[\tilde{\tilde{t}} \overline{\tilde{t}}] \quad \tilde{\bar{t}}$ stoponium $\gamma e \rightarrow \tilde{e}^{-} \tilde{\chi}_{1}^{0} \quad M_{\tilde{e}^{-}}<0.9 \times 2 E_{0}-M_{\tilde{\chi}_{1}^{0}}$ $\gamma e \rightarrow \tilde{\nu}_{e} \tilde{\chi}_{1}^{ \pm} \rightarrow \tilde{\nu}_{e} \tilde{\nu}_{\mu} \mu \quad$ sneutrino production $\gamma e \rightarrow \nu W^{+} \rightarrow W^{+} W^{-} l^{+} \quad$ Majorana neutrino $\gamma \gamma \rightarrow W^{+} W^{-} \quad$ anom. $W$ inter., extra dim. $\gamma e^{-} \rightarrow W^{-} \nu_{e} \quad$ anom. $W$ couplings $\gamma \gamma \rightarrow W W+W W(Z Z)$ strong $W W$ scattering $\gamma \gamma \rightarrow t \bar{t} \quad$ anom. $t$-quark interact. $\gamma e^{-} \rightarrow \bar{t} b \nu_{e}$ $\gamma \gamma \rightarrow$ hadrons $\gamma e^{-} \rightarrow e^{-} X$ and $\nu_{e} X$ $\gamma g \rightarrow q \bar{q}, c \bar{c}$ $\gamma \gamma \rightarrow J / \psi J / \psi$ struct. functions gluon distr. in the photon QCD Pomeron

Table 1: Gold-plated processes at PLC

$\gamma \gamma$ colliders can be found in reviews [7, 45, 46, 47, 48, 49, 50, 51, references therein, and many other papers.

So, the physics reaches of a $\gamma \gamma, \gamma e$ and $e^{+} e^{-}$colliders are comparable. The only advantage of $e^{+} e^{-}$collisions is the narrower luminosity spectrum, the feature that is useful but not obligatory. Also, the hadronic background ( $\gamma \gamma \rightarrow$ hadrons $)$ at the photon collider would be several times greater. In $e^{+} e^{-}$collisions, the beams at the IP also contain many beamstrahlung and virtual photons that produce hadrons.

The photon collider can be added to the linear $e^{+} e^{-}$collider at a very small incremental cost. The laser system and modification of the IP and one of the detectors would add about $3-4 \%$ to the total ILC cost. Some decrease of the $e^{+} e^{-}$running time is a negligible price to pay for the opportunity to look for new phenomena in other types of interactions.

\section{Summary}

The physics expected in the $0.1-1 \mathrm{TeV}$ region is very exciting, and the ILC is a unique machine for the study physics in this energy region. However, it is a very expensive machine, and therefore it should strive to achieve ultimate performance and get maximum results. Answers to the mysteries of the origin of mass and the nature of the dark matter in the Universe would be a triumph of the entire mankind and would give excitement to several generations; from this perspective, $\$ 10 \mathrm{~B}$ or even $\$ 30 \mathrm{~B}$ is a negligible price to pay for these breakthroughs in human understanding of the Universe.

There is a very good chance that a linear collider will be built somewhere in the world, and then the photon collider will inevitably happen, and the study of new phenomena in $e^{+} e^{-}, e^{-} e^{-}, \gamma \gamma, \gamma e$ will bring us to a new level of understand the world we live in!

\section{References}

[1] V.I. Telnov, talk at Photon2007, Paris 9-13, 2007, slides of this paper: http://indico.cern.ch/getFile.py/ access? contribId=67\&session $I d=10 \&$ res $I d=0 \&$ materialId=slides\&conf $I d=3841$

[2] I.F. Ginzburg, G.L. Kotkin, V.G. Serbo, and V.I. Telnov, Pizma ZhETF, 34514 (1981); [JETP Lett. 34, 491 (1982)]. 
[3] I.F. Ginzburg, G.L. Kotkin, V.G. Serbo, V.I. Telnov, Nucl. Instrum. Meth. 205, 47 (1983); I.F. Ginzburg, G.L. Kotkin, S.L. Panfil, V.G. Serbo, and V.I. Telnov. Nucl. Inst. Meth., A219, 5 (1984).

[4] V.I. Telnov, Nucl. Instrum. Meth.,A294, 72 (1990).

[5] V.I. Telnov, Nucl. Instrum. Meth. A355, 3 (1995).

[6] V.I. Telnov, Acta Physica Polonica, B37, 633 (2006), physics/0602172 slides: http://indico.cern.ch/materialDisplay.py? subContId=3\&contribId=s1t3\&sessionId $=1 \&$ material $I d=0$ \& $\operatorname{conf} I d=a 053282$

[7] B. Badelek et. al.,[ECFA/DESY Photon Collider working group], Intern. Journ. Mod. Phys. A30, 5097 (2004), hep-ex/0108012

[8] V.I. Telnov, Acta Physica Polonica, B37, 1049 (2006); physics/0604108 slides: http://indico.cern.ch/materialDisplay.py? subCont Id=1\&contribId=s5t 4 \&session Id $=5 \&$ material $I d=0$ \& $\operatorname{conf} I d=a 053282$

[9] The NLC Design Group, Zeroth-Order Design Report for the NLC, LBNL-5424, SLAC-474, UCBL-ID-124161, UC-414, SLAC, 1996.

[10] N. Akasaka et al, JLC Design Study, KEKREPORT-97-1;

I. Watanabe, et al, KEK-REPORT-97-17.

[11] R. Brinkmann et al, Nucl. Instr. Meth., A406, 13 (1998), hep-ex/9707017.

[12] ILC Reference Design Report, August 2007, ILC-Report-2007-001;

http://www.linearcollider.org/cms/ ?pid $=1000025$

[13] ILC Interaction Region Engineering Design Workshop, IRENG07, Sept.17-21,SLAC, Stanford, USA;

http://www-conf.slac.stanford.edu/ireng07/

[14] F. R. Arutyunian and V. A. Tumanian, Phys.Lett. 4, 176 (1963); F. R. Arutyunian I.I. Goldman and V. A. Tumanian, Zh. Eksp. Teor. Fiz. (USSR) 45, 312 (1963);

R. H. Milburn, Phys. Rev. Lett. 10, 75 (1963).

[15] M. Galynskii, E. Kuraev, M. Levchuk, and V. I. Telnov, Nucl. Instrum. Meth., A472, 267 (2001), hep-ph/0012338

[16] V.I. Telnov, Proc. of 2005 Intern. Linear Collider Physics and Detector Workshop and 2nd ILC Acceler. Workshop, Snowmass, Colorado, 14-27 Aug 2005, ECONF C0508141:PLEN0020,2005, physics/0512048 Slides: http://www.slac.stanford.edu/econf/ C0508141/proc/pres/PLEN0020_TALK.PDF
Paper: http://www.slac.stanford.edu/econf /C0508141/proc/papers/PLEN0020.PDF

[17] G. Klemz, K. Monig and I. Will, Nucl. Instrum. Meth. A564, 212 (2006), physics/0507078

[18] V.I. Telnov, In Physics and Experiments with Linear Colliders, Saariselka, Finland, 1992, v2, pp. 739-775, Ed. R. Orava et al., World Scientific.

[19] V.I. Telnov, talk at the ECFA workshop on linear colliders, Montpellier, France, 12-16 November 2003; slides: http://www-h1.desy.de/ ${ }^{m a x f i e l d / g g c o l / m o n t ~}$ pellier_talks/Valery_lumispec_MONT1.PDF

[20] V.I. Telnov, LCWS-2005-1113, Jul 2005. 6pp. Proceedings of 2005 International Linear Collider Workshop (LCWS 2005), Stanford, California, 18-22 Mar 2005, pp 1113, e-Print: physics/0507134

[21] L.I. Shekhtman and V.I. Telnov, Proc. of Intern. Conf. on Linear Colliders (LCWS 04), Paris, France, 19-24 Apr 2004, Paris 2004, Linear colliders, v1, p.507; physics/0411253.

[22] A. Seryi, talk at the LCWS06, 9-13 March 2006, Bangalore, India; slides: http://indico.cern.ch/getFile.py/access? contribId=207\&sessionId=11\&res Id=1\&mater ialld=slides\& $\operatorname{conf} \mathrm{Id}=568$

[23] V.I. Telnov, Proc. LCWS06, India, March 2006. To be published in Pramana Journal of Physics, v. 69, No.6, Dec. 2007, physics/0610287

[24] V.I. Telnov, talk at the LCWS07, Hamburg, May 27-June 4, 2007; slides: http://ilcagenda.linearcollider.org/get File.py/access? contribId $=446 \&$ sessionId $=$ 79\&res Id=2\&material Id=slides\&conf Id=1296

[25] J. Gronberg, talk at the LCWS07, Hamburg, May 27-June 4, 2007; slides: http://ilcagenda.linearcollider.org/get File. py/access? contribId $=459$ \&sessionId= 148\&res Id=1\&material Id=slides\&conf $I d=1296$

[26] V.I.Telnov, talks at the ILC Interaction Region Engineering Design Workshop, IRENG07, Sept.17-21,SLAC, Stanford, USA; slides: http://ilcagenda.linearcollider.org/getFi le.py/access? contribId=81\&amp; sessionId= 13\&amp; res Id=1\&amp; material Id=slides\&amp; conf $I d=2169$.

[27] P. Chen and V. I. Telnov, Phys. Rev. Lett., 63, 1796 (1989).

[28] V.I. Telnov, Proc. of Workshop 'Photon 95', Sheffield, UK, April 8-13, 1995, Ed. D.Miller et al., World Scientific, 1995, p.369;

V.I. Telnov. In Proc. of ITP Workshop "Future High Energy Colliders", Santa Barbara, CA, USA, 1996, AIP Conf. Proc. 397, 259 (1997), physics/9706003 
[29] V.I. Telnov, Nucl. Instrum. Meth., A472, 43 (2001), hep-ex/0010033

[30] V.I. Telnov, Phys. Rev. Lett.,78, 4757 (1997), Erratum: Phys. Rev. Lett. 80, 2747 (1998), hep-ex/9610008

V.I. Telnov, Nucl. Instrum. Meth., A455, 63 (2000), hep-ex/0001029

[31] V.I. Telnov, Requirements to beam sizes at photon colliders and problems of realization; http://icfa-nanobeam.web.cern. ch/icfananobeam/slides/telnov_gg.pdf \protect \vrul nanobeam.web.cern.ch/icfa-nanobeam/slides/ telnov_gg.pdf

[32] A. Seryi, Proc. of 2005 Intern. Linear Collider Physics and Detector Workshop and 2nd ILC Acceler. Workshop, Snowmass, Colorado, 14-27 Aug 2005, ECONF C0508141:ILCAW120,2005, http://www.slac.stanford.edu/econf/ C0508141/proc/pres/ILCAW1203_TALK.PDF

[33] A.V. Pak, D.V. Pavluchenko, S.S. Petrosyan, V.G. Serbo and V.I. Telnov, Nucl. Phys. Proc. Suppl., 126, 379 (2004), hep-ex/0301037

[34] G.V. Jikia, Nucl. Phys.B412, 57 (1994); R. Belusevic and G. Jikia, Phys. Rev. D70, 073017 (2004).

[35] V.I. Telnov, Proc. LCWS06, India, March 2006. To be published in Pramana Journal of Physics, v. 69, No.6, Dec. 2007, physics/0610285

[36] V.I. Telnov. In Workshop on Physics and Exper. with Linear $e^{+} e^{-}$Colliders, Waikoloa, USA, p. 323, 1993. World Scientific.

[37] V. Makarenko, K. Monig and T. Shishkina, Eur. Phys. J. C 32, (2003) SUPPL1143, hep-ph/0306135

[38] D. Strickland and G. Mourou, Opt. Commun., 56, 219 (1985). M. Perry and G. Mourou, Science, 264, (1994) 917.

[39] V. Telnov, Optics at the interaction region of photon colliders, Talk at the Int. meeting on Gamma-Gamma Collider, Hiroshima, Japan, Feb. 10-12, 1999; slides:

http://photon.hepl.hiroshima-u.ac.jp/ $\mathrm{lab} / \mathrm{jlc} / \mathrm{mini}$-ws/agenda.html

[40] V.I, Telnov, Nucl. Phys. Proc. Suppl. 82, 359 (2000), hep-ex/9908005

[41] V.I. Telnov, Int. J. of Mod. Phys. A15, 2577 (2000), hep-ex/0003024

[42] I. Will, T. Quast, H. Redlin and W. Sandner, Nucl. Instrum. Meth. A472, 79 (2001).

[43] Proc. Intern. Workshop On High-Energy Photon Colliders (GG 2000), Hamburg, Germany, June 14-17, 2000, ed. R.D. Heuer, V.I. Telnov, N.J. Walker, Nucl. Instrum. Meth. A472, 1-321 (2001).
[44] V.Zomer, talk at Photon 2007, Towards a polarized positron source, Paris 9-13, 2007, these proceedings; slides:

http://indico.cern.ch/getFile.py/access? contribId=70\&sessionId=25\&res Id=1\&materi alId=slides\&conf $I d=3841$

[45] E.E. Boos, Nucl. Instrum. Meth., A472, 22 (2001), hep-ph/0009100

[46] M. M. Velasco et al., Proc. of the $A P S / D P F / D P B$ Summer Study on the Future

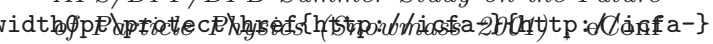
C010630 (2001) E3005, hep-ex/0111055

[47] A. De Roeck, DESY-04-123GF, Nov 2003, hep-ph/0311138

[48] M. Krawczyk, Eur. Phys. J. C33, S638 (2004), hep-ph/0312341

[49] S.J. Brodsky, Int. J. Mod. Phys. A20, 7306 (2005);

S. Brodsky, Acta Physica Polonica B37, 619 (2006).

[50] M. M. Muhlleitner and P. M. Zerwas, Acta Physica Polonica, B37, 1021 (2006), hep-ph/0511339

[51] F. Bechtel, G. Klamke, G. Klemz, K. Monig, H. Nieto, H. Nowak, A. Rosca, J. Sekaric, A. Stahl, Nucl. Instrum. Meth., A564, 243 (2006), physics/0601204 